\title{
SISTEMA DE OTTO-CODIFICAÇÃO MODIFICADO PARA ENDEREÇAMENTO DE REDES HIDROGRÁFICAS ${ }^{1}$
}

\author{
Natanael dos Santos Silva ${ }^{2}$, Carlos Antonio Alvares Soares Ribeiro², Wenderson Ribeiro Barroso ${ }^{3}$, Paula \\ Euclides Álvares Ribeiro ${ }^{4}$, Vicente Paulo Soares ${ }^{2}$ e Elias Silva ${ }^{2}$
}

\begin{abstract}
RESUMO - Há, no âmbito do gerenciamento de recursos hídricos, uma carência por sistemas de aplicação global que referenciem, indiquem e identifiquem, de forma única e eficiente, a organização espacial das bacias hidrográficas e respectivas redes de drenagem. Neste trabalho, propôs-se uma modificação no sistema de endereçamento originalmente concebido por Otto Pfafstetter. A nova metodologia utiliza tão-somente as redes de drenagem no formato vetorial e considera, em vez da área das bacias, o comprimento dos seus cursos d'água para codificálos. Essa estratégia elimina o elevado ônus associado à obtenção de dados de altimetria e à derivação dos respectivos modelos digitais de elevação, imprescindíveis à correta determinação das áreas de contribuição das bacias hidrográficas. Adicionalmente, elimina-se o esforço computacional das operações baseadas em localização espacial, uma vez que agora é possível realizá-las exclusivamente por atributos. Com base nessa metodologia, desenvolveuse o aplicativo Otto-Sys, utilizando a linguagem de programação AML, nativa do sistema de informações geográficas Arc/INFO workstation. Para exemplificar os resultados da otto-codificação por comprimento dos cursos d'água, apresentou-se um estudo de caso utilizando a rede de drenagem do rio Caeté, afluente do rio Iaco, pertencente à bacia do rio Purus, no Estado do Amazonas. O detalhamento dessa hidrografia vetorial implicou cinco níveis de codificação. Tendo-se codificado toda a rede hidrográfica, qualquer sistema de informações geográficas, mesmo aqueles que não dispõem de recursos específicos para análises e navegação em redes, poderá oferecer serviços de navegação topológica, organização, estruturação, endereçamento e gerenciamento dessas bases de dados. Desse modo, o acesso em tempo real a tais procedimentos poderá ser perfeitamente estendido a qualquer dispositivo capaz de acessar os sistemas web.
\end{abstract}

Palavras-chave: Otto-codificação, endereçamento de redes hidrográficas e topologia de redes.

\section{AN IMPROVED STREAM NETWORK ADDRESSING SYSTEM: THE MODIFIED PFAFSTETTER CODING SCHEME}

\begin{abstract}
This paper presents a new numbering system based on the Pfafstetter codification scheme developed for stream networks. Its main advantage is the use of the segments' length instead of the catchments' areas for establishing the Pfafstetter code, therefore eliminating the need for elevation data. This methodology was then implemented in a software named Otto-Sys, developed for Arc/INFO workstation in AML. In order to present the results of this new approach, a case study was carried out for the stream network of the Caeté River, a tributary of Iaco River, belonging to the Amazon River basin. The details of such a digital hydrographic dataset led to 5 level-deep codes. Each codification level is stored as a new column of the attribute table and the corresponding level dictates the number of digits of the code. Once this codification scheme has been established, network-tracing tasks can be performed in any geographic information system using only attribute queries. This strategy speeds up such processes enabling them to be extended to real-time web-based devices.
\end{abstract}

Keywords: Pfafstetter system, stream network numbering systems and network topology.

\footnotetext{
${ }^{1}$ Recebido em 13.02.2007 e aceito para publicação em 22.08.2008.

${ }^{2}$ Departamento de Emgenharia Florestal da Universidade Federal de Viçosa (UFV). E-mail:<cribeiro@ufv.br>.

${ }^{3}$ Graduado em Engenharia de Agrimensura pela UFV. E-mail:<natanss@ gmail.com>

${ }^{4}$ Graduanda em Engenharia Aeronáutica pela Universidade de São Paulo (USP). E-mail:<pearibeiro@ yahoo.com.br>
} 


\section{INTRODUÇÃO}

A gestão de bacias hidrográficas é estratégica para a utilização racional e integrada dos recursos hídricos. A Lei Federal no 9433, promulgada em 8 de janeiro de 1997, define a bacia hidrográfica como a unidade territorial para sua implementação e para a atuação do Sistema Nacional de Gerenciamento de Recursos Hídricos (SNGRH). A Conferência das Águas e Meio Ambiente, em Dublin, e a Conferência das Nações Unidas sobre Meio Ambiente e Desenvolvimento em 1992 (Rio 92), no Rio de Janeiro, reafirmaram a importância de se determinarem unidades administrativas para o desenvolvimento, planejamento e gerenciamento integrado dos recursos hídricos.

Há, no entanto, uma carência de sistemas de aplicação global que referenciem, indiquem e identifiquem, de forma única e eficientemente, a natureza das bacias hidrográficas (TONELLO et al., 2006; VERDIN e VERDIN, 1999). Tendo por base a topografia das bacias de contribuição e a topologia das respectivas redes de drenagem, Otto Pfafstetter, engenheiro do extinto Departamento Nacional de Obras e Saneamento (DNOS), desenvolveu, em 1989, um sistema de endereçamento auto-replicável e extremamente simples (PFAFSTETTER, 1989; SILVA, 1999). Verdin e Verdin (1999) implementaramno em um sistema de informações geográficas, possibilitando a estruturação, armazenamento e recuperação de dados em bases hidrográficas. Porquanto a malha hidrográfica brasileira já esteja disponível (http://www.ibge.gov.br/home/geociencias/ default prod.shtm\#TOPO) em formato digital vetorial, para diversas escalas, o mesmo ainda não ocorre com relação aos modelos digitais de elevação das respectivas bacias. Tais modelos são imprescindíveis para se automatizarem os processos de delineamento de bacias hidrográficas e a correta determinação de suas áreas de contribuição, condição básica - e um dos principais impedimentos - para se aplicar a otto-codificação (RIBEIRO et al., 2005). Um Sistema de Informações Hidrológicas (SIH) que integre e modele tais variáveis requer, necessariamente, soluções baseadas em estrutura vetorial de redes que referenciem, através de um mecanismo de endereçamento linear, cada segmento da hidrografia. O uso de modelos digitais de elevação nesse contexto seria não apenas desnecessário, mas também oneroso para um projeto dessa natureza.
Para transpor os obstáculos mencionados, investigou-se, neste trabalho, a utilização do comprimento dos cursos d'água - em vez da área das bacias - como base para se efetuar a otto-codificação, implementandoa em um sistema de informações geográficas comercial. O objetivo dessa nova abordagem é eliminar, por completo, o uso de modelos digitais de elevação, possibilitando lidar tão-somente com a rede de drenagem no formato vetorial, porém preservando-se a eficiência do sistema original de otto-codificação.

\section{MATERIAL E MÉTODOS}

Tendo em vista a disponibilidade de informações digitais de hidrografia já produzidas no âmbito do Convênio Projeto SIVAM/Universidade Federal de Viçosa e considerando a uniformidade da densidade de drenagem, que é bastante variável em um mesmo conjunto de cartas do IBGE, optou-se por desenvolver este estudo de caso em uma área típica da depressão da Amazônia Ocidental. Especificamente, para ilustrar os resultados da metodologia proposta para efetuar a otto-codificação, selecionou-se a rede hidrográfica a montante da estação hidrológica Seringal Guarany, localizada no rio Caeté, afluente do rio Iaco, sub-bacia do rio Purus, conforme mostra a Figura 1. Essa área de estudo, com uma superfície de $3.516,43 \mathrm{~km}^{2}$, localiza-se entre os paralelos $9^{\circ} 08^{\prime}$ e $10^{\circ} 03^{\prime}$ de latitude sul e entre os meridianos de $69^{\circ}$ $22^{\prime}$ e $70^{\circ} 04^{\prime}$ de longitude $\mathrm{WGr}$, com altitudes variando entre $170 \mathrm{~m}$ e $320 \mathrm{~m}$ e apresentando densidade de drenagem igual a $0,5 \mathrm{~km} / \mathrm{km}^{2}$.

\subsection{Sistema de otto-codificação}

No sistema de endereçamento proposto por Otto Pfafstetter, a importância de qualquer rio está diretamente relacionada à área de sua bacia hidrográfica. A ottocodificação destaca-se pela representação fidedigna da estruturação inerentemente recursiva das bacias hidrográficas, por meio de utilização de códigos. Para tanto, faz uso do sistema decimal de numeração, a fim de estabelecer uma relação topológica entre cada par de segmentos ou entre um segmento e a respectiva foz da rede. Quanto maior o valor do código do tributário analisado, mais distante ele estará da foz do curso principal ao qual se conecta diretamente. Conseqüentemente, para dois tributários de um mesmo curso principal, aquele que tiver o código de maior valor estará mais 
próximo da sua nascente. Há, ainda, uma distinção entre os dígitos pares e ímpares de um código, permitindose saber se o segmento pertence ou não ao curso principal. Uma importante vantagem dessa estratégia é o uso inteligente e eficiente de dígitos: utilizam-se menos dígitos que os sistemas similares usuais, e os códigos são infinitamente replicáveis, i.e., não se impõem quaisquer restrições ao número de dígitos necessários para endereçar, de maneira única, qualquer segmento da rede de drenagem, independentemente do nível de detalhamento desta rede ou de sua abrangência (FURNANS e OLIVERA, 2001).

No sistema de otto-codificação original, a diferenciação entre rio principal e tributário é feita usandose a respectiva área de drenagem como variável de decisão. Assim, em qualquer confluência o rio principal é aquele que possui a maior área de drenagem nos dois segmentos analisados.

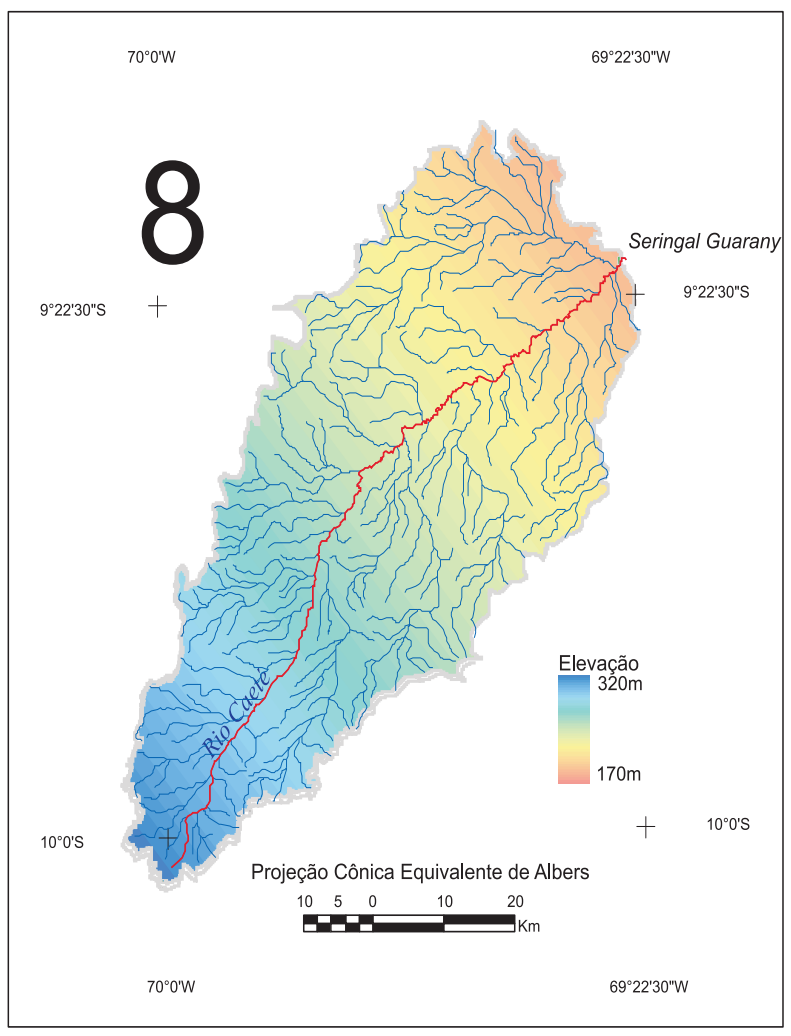

Figura 1 - Rede de drenagem de estudo, situada a montante da Estação Fluviométrica Seringal Guarany.

Figure 1 -Stream network located upstream of the Seringal Guarany stream gage station, and used as a case study.

\subsection{Modificando o sistema de otto-codificação}

Inúmeros eventos ocorrem exclusivamente ao longo da hidrografia e, portanto, requerem soluções baseadas em uma estrutura vetorial de redes que os referenciem, através de um sistema de endereçamento apropriado. Entre esses, destacam-se: a localização de estações fluviométricas, de usinas hidrelétricas, de pontos batimétricos; os cálculos de vazão d'água e da respectiva fração outorgável para pontos específicos; a capacidade de autodepuração de um caudal; a identificação da planície de inundação para determinada cota do rio; etc.

O sistema ora proposto se baseia no conhecido método de codificação de Pfafstetter e carrega em si todas as suas vantagens. Enquanto o sistema original requer tanto a rede hidrográfica quanto a área drenada por cada segmento como parâmetro para se definirem os quatro maiores tributários do curso principal, a metodologia aqui proposta utiliza tão-somente o comprimento dos tributários como variável de decisão. Isso elimina o considerável ônus da obtenção de dados de altimetria e da geração de modelos digitais de elevação, imprescindíveis à obtenção acurada das áreas de drenagem, que delongam, sobremaneira - e algumas vezes até mesmo impedem -, a condução do processo de endereçamento (DJOKIC e YE, 2000).

\subsection{Estrutura e implementação do sistema computacional}

A estruturação lógica do sistema, conforme ilustra a Figura 2, constitui-se de quatro módulos, a saber: i) módulo de identificação do maior curso d'água da rede hidrográfica, ii) módulo de identificação dos (quatro ou menos) maiores tributários do maior curso, iii) módulo de codificação dos segmentos de otto-bacias e iv) módulo de codificação dos segmentos de interbacias.

A implementação dos procedimentos presentes em cada módulo foi feita em AML (Arc Macro Language), linguagem nativa do sistema de informações geográficas Arc/INFO, versão 8.3, para Windows XP.

O primeiro módulo requer, como dados de entrada, a hidrografia vetorial unifilar ${ }^{6}$ e orientada no sentido do escoamento, e o código identificador da foz da subrede em análise. Para identificação do maior curso, fezse um percurso da rede rio acima, acumulando-se o comprimento de cada segmento. Em cada segmento,

${ }^{6}$ Trechos da hidrografia com largura suficiente para serem representados com margem dupla deverão ser substituídos pelas respectivas linhas de centro.

R. Árvore, Viçosa-MG, v.32, n.5, p.891-897, 2008 
esse valor acumulado representa a sua distância em relação à foz. Isso feito, selecionou-se o segmento mais distante e realizou-se um segundo caminhamento, agora em sentido descendente, selecionando os arcos que compõem o trajeto até a foz. O maior curso é denotado pelo conjunto de arcos que constituem esse caminho, desde a nascente até a foz. Na Figura 3 mostram-se os sentidos de caminhamentos para a) o assinalamento da distância de cada segmento à foz e b) a delimitação do maior curso da hidrográfica analisada.

- De posse dos atributos Hidro (identificador da rede), NumOtto (número de tributários) e Maior (conjunto de arcos que compõem o maior curso), o módulo Quatro é, então, utilizado para localizar e armazenar, em ordem crescente, os identificadores (IDs) dos quatro maiores tributários, em uma estrutura de dados linear (Lista4) que permanece guardada na memória principal enquanto o programa estiver em execução. Essa lista de identificadores será posteriormente utilizada pelo módulo de ottocodificação.

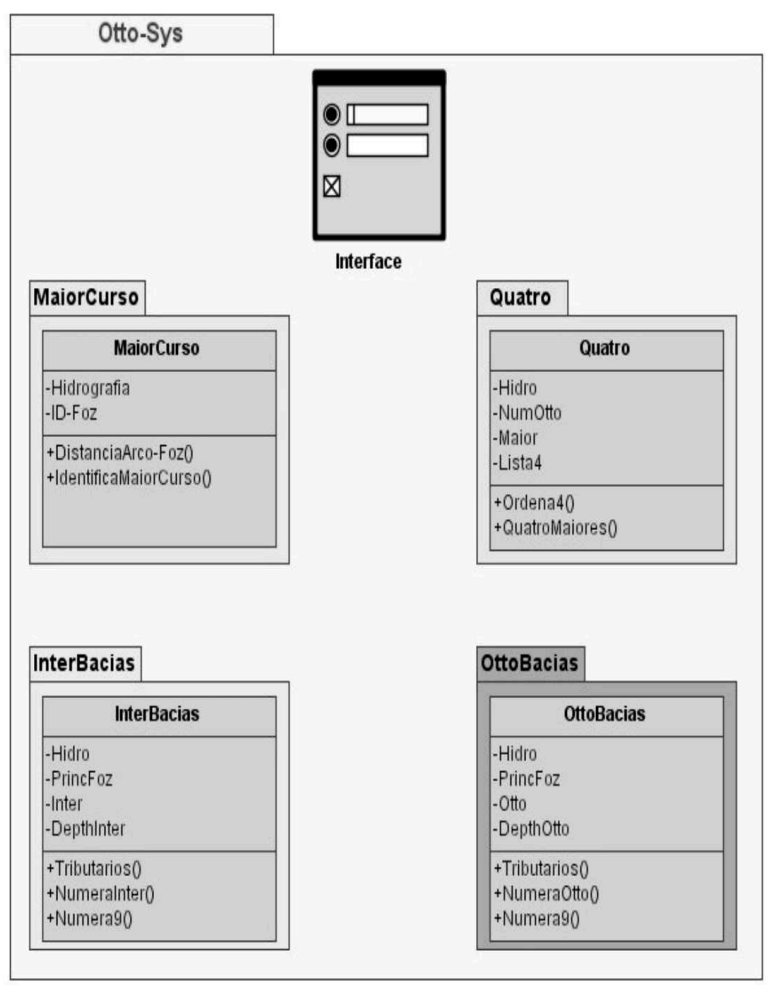

Figura 2 - Estrutura conceitual do sistema Otto-Sys. Figure 2-Conceptual framework of the system used.

R. Árvore, Viçosa-MG, v.32, n.5, p.891-897, 2008

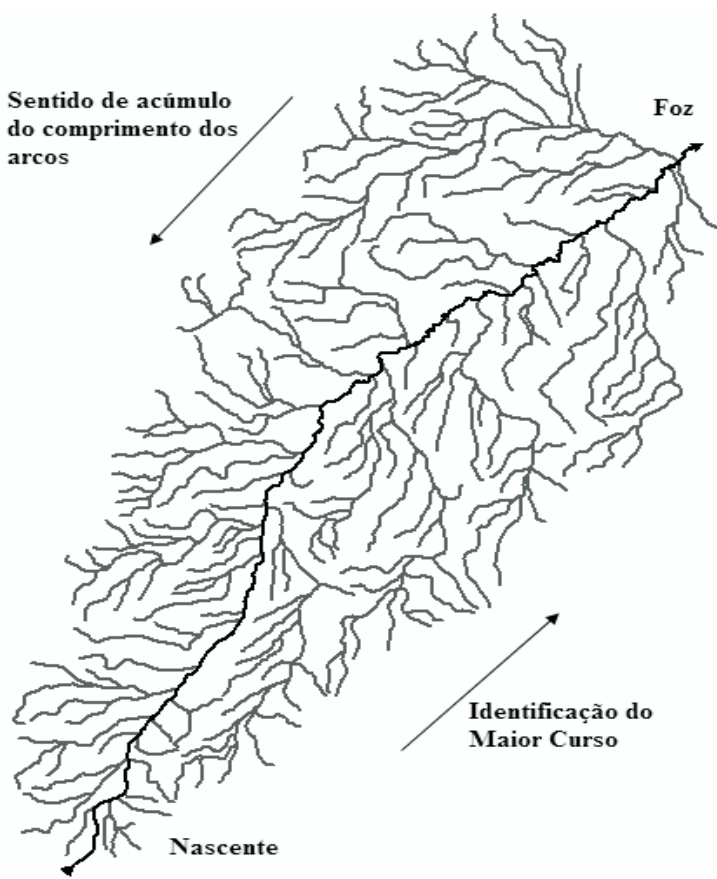

Figura 3 - Sentido de acúmulo do comprimento dos arcos para delimitação do maior curso de uma rede de drenagem.

Figure 3 -Finding the main stem via stream network tracing.

- OttoBacias é o módulo de otto-codificação propriamente dito. Inicialmente, identificam-se as confluências dos maiores tributários (no máximo quatro) com o curso d'água principal e individualizam-se as respectivas redes de segmentos a montante de cada uma dessas confluências. A codificação das sub-redes dos tributários dá-se no sentido da foz da bacia para a nascente do maior curso, aplicando-se recursivamente as rotinas do módulo OttoBacias até que não haja mais tributários para o segmento analisado. Essa regra de parada caracteriza um arco associado a um rio de cabeceira.

- O trecho da rede hidrográfica compreendido entre duas confluências consecutivas dos maiores tributários com o curso principal é denominado interbacia. A identificação dos arcos das interbacias do maior curso dá-se também no sentido rio acima e é feita no módulo InterBacias. Para fins de endereçamento, os arcos de cada interbacia são tratados como uma rede hidrográfica isolada, que goza das mesmas propriedades e comportamentos da rede de entrada, e, portanto, o sistema chamará recursivamente as rotinas do módulo OttoBacias para realizar a codificação dos tributários desse trecho do curso d'água principal. 
- Por fim, o módulo de interface encapsula, de forma prática e amigável, a funcionalidade do sistema, além de controlar o fluxo de chamada das rotinas dos outros módulos.

\section{RESULTADOS E DISCUSSÃO}

Neste exemplo de aplicação, apresentam-se as principais interfaces do sistema Otto-Sys, bem como os passos necessários para a codificação completa da rede. O ponto de partida da codificação é a especificação da rede hidrográfica vetorial unifilar. Em seguida, verifica-se a qualidade dessa rede de drenagem quanto à conectividade de seus arcos e unicidade da foz e ao sentido do escoamento de seus segmentos. Se qualquer um desses requisitos não for atendido, a execução do programa é interrompida, notificandose o usuário. Na Figura 4 observa-se a interface principal do sistema com os componentes que dão acesso à seleção, verificação e codificação da rede hidrográfica.

É possível salvar em disco um relatório de execução do programa contendo informações do tempo de execução, número de segmentos codificados, níveis de codificação atingidos e eventuais inconsistências na base de dados, conforme ilustra a Figura 5.

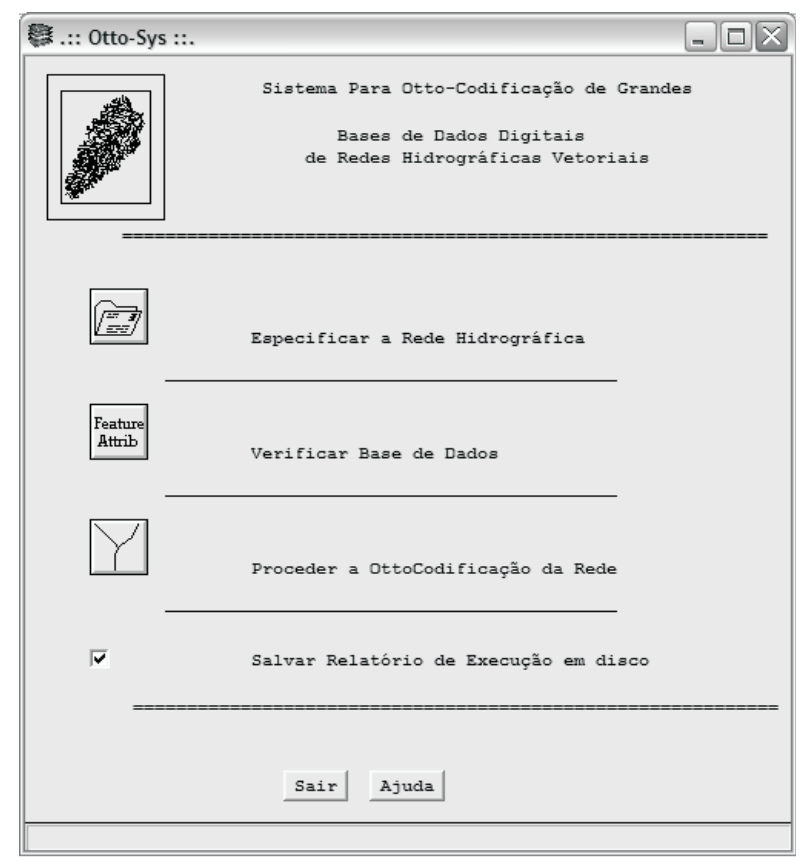

Figura 4 - Principais componentes da interface do sistema Otto-Sys.

Figure 4-Otto-Sys user's main interface components.

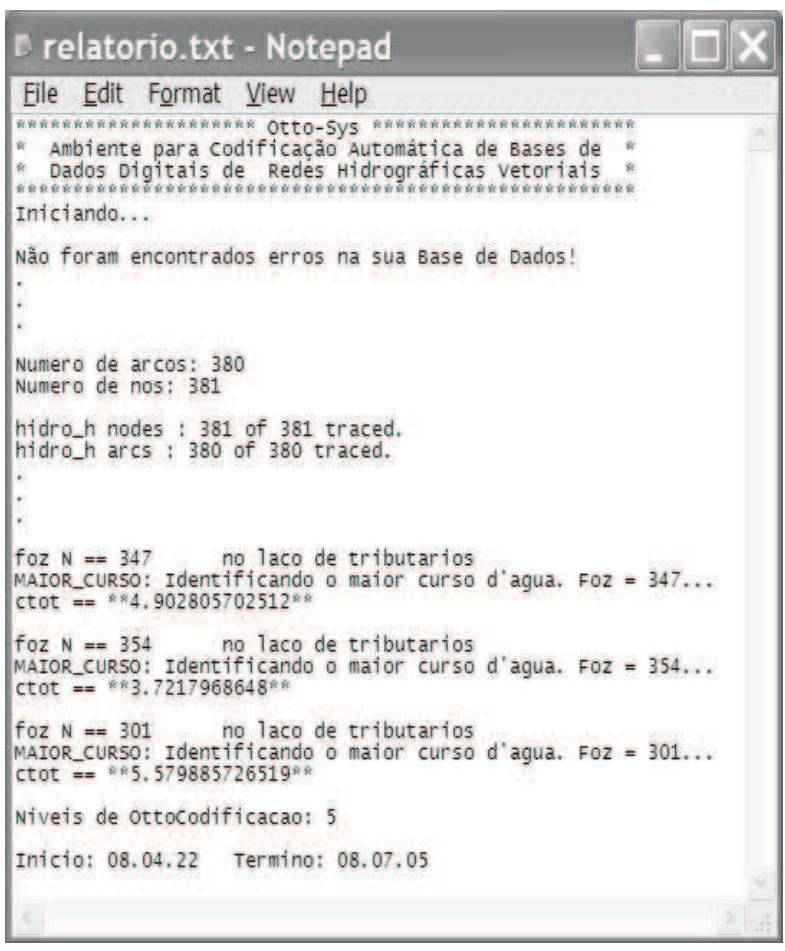

Figura 5 - Relatório típico da execução de uma Otto-codificação. Figure 5 - Typical report of a Pfafstetter-codification run.

A codificação inicia-se pelo primeiro segmento conectado diretamente à foz da rede. No presente caso, adotou-se, arbitrariamente, o nível 1 para início da otto-codificação. Em cada novo nível de codificação, adiciona-se um campo na tabela de atributos da hidrografia até que a condição de parada da recursividade seja satisfeita. Ao final do processamento, ter-se-ão os campos OTTO_BACIA, contendo o código completo de identificação do segmento, mais os campos OTTO 1 , OTTO2,..., OTTOn, em que n é o último nível de codificação atingida. Veja na Figura 6 a tabela de atributos resultante da execução do programa. No registro selecionado, tem-se o código completo do segmento associado, assim como os códigos dos seus cinco níveis de otto-codificação.

Embora requeira mais espaço, o armazenamento dos códigos de cada nível tem a vantagem de agilizar, sobremaneira, o processo de consulta aos dados para qualquer nível desejado. A Figura 7 ilustra a organização dos arcos da rede para o nível 1 de codificação.

R. Árvore, Viçosa-MG, v.32, n.5, p.891-897, 2008 


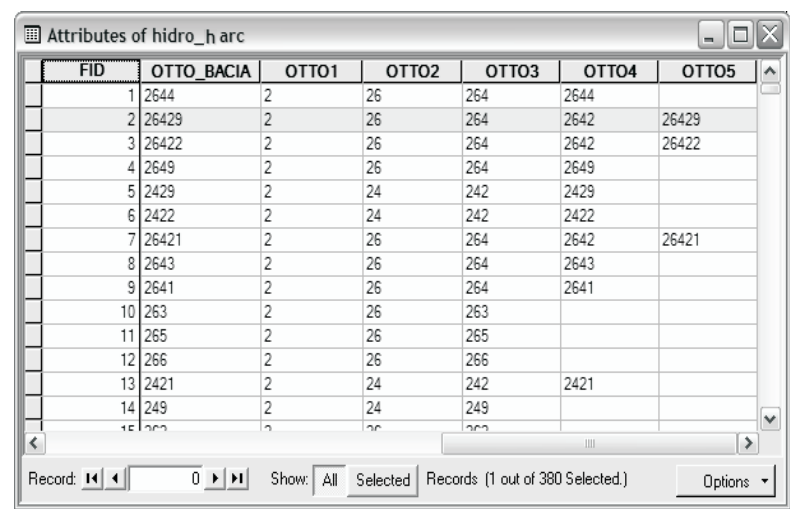

Figura 6 - Tabela de atributos da rede de drenagem codificada. Para cada segmento, tem-se o seu código completo, bem como os dos respectivos níveis de ottocodificação atingidos.

Figure 6 - Stream network attribute table showing the full codes and all the other corresponding Pfafstettercodification levels.

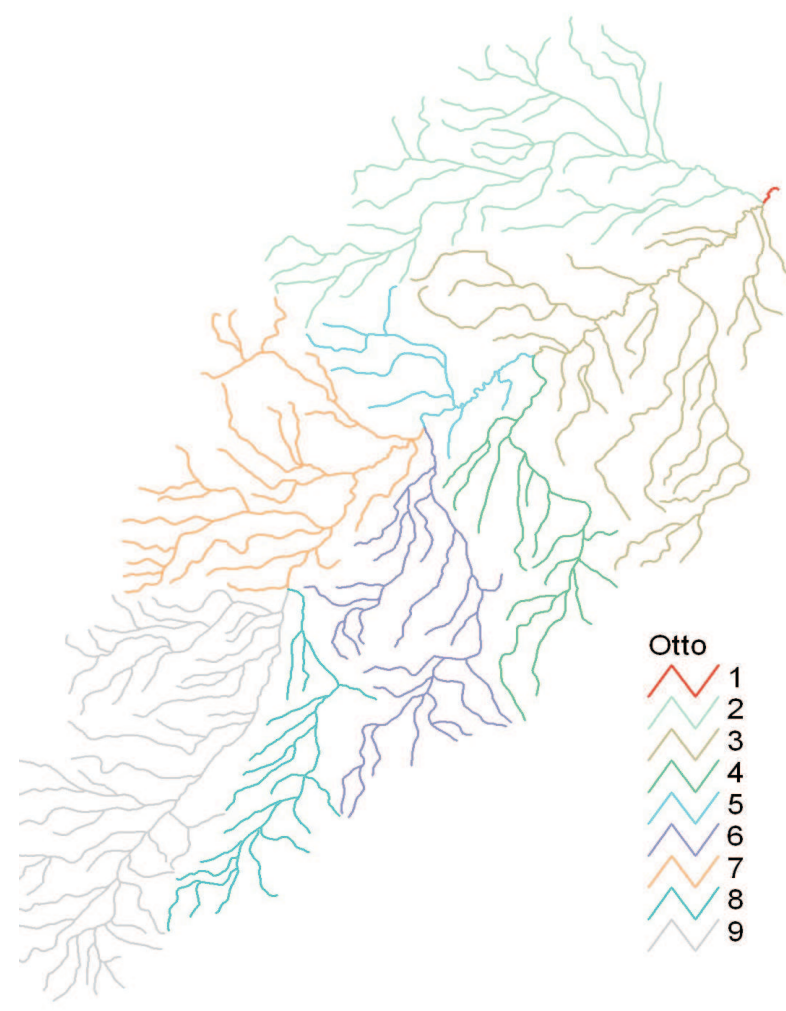

Figura 7 - Primeiro nível de otto-codificação de uma rede de drenagem com base nos comprimentos dos seus segmentos.

Figure 7 - First level-deep Pfafstetter code for a given stream network. The codification scheme was established based on the length of its segments.

\section{CONCLUSÕES}

O sistema proposto destaca-se pela sua eficiência em endereçar todo e qualquer segmento de uma rede hidrográfica vetorial, independentemente de seu tamanho ou nível de detalhamento. Por basear-se nos princípios de codificação de Otto Pfafstetter, a geração e atribuição de códigos são feitas de forma robusta e inteligente, descrevendo, com fidelidade, a estrutura naturalmente aninhada das bacias e de suas redes de drenagem.

Uma vez que todas as informações necessárias à otto-codificação modificada encontram-se disponíveis na própria topologia da rede hidrográfica, dispensa-se completamente o uso dos modelos digitais de elevação para tais fins, simplificando a análise de uma multitude de eventos e parâmetros hidrológicos dispostos ao longo dos cursos d'água. A navegação topológica de uma base de dados assim estruturada poderá, então, ser plenamente executada mesmo em sistemas de informações geográficas que não disponham de recursos específicos para análises e navegação em redes.

O desenvolvimento e implementação dessa metodologia, sob a forma de um software (Otto-Sys), representam considerável avanço e solucionam as principais limitações dos métodos atuais de organização, endereçamento, gerenciamento e disponibilização de grandes bases de dados de redes hidrográficas, viabilizando-se a execução de tais procedimentos em tempo real em computadores de desempenho razoável. Criam-se, assim, as condições necessárias para o oferecimento desses serviços via internet.

\section{AGRADECIMENTOS}

Ao CNPq, pela concessão da bolsa de produtividade em pesquisa e pelas bolsas de iniciação científica.

\section{REFERÊNCIAS}

DJOKIC, D.; YE, Z. DEM preprocessing for efficient watershed delineation. In: Maidment, D.; Djokic, D. (Eds.).Hydrologic and hydraulic modeling support with geographic information systems. Redlands: Environmental Systems Research Institute, 2000. p.65-84. 
FURNANS, F.; OLIVERA, F. Watershed topology: The Pfafstetter system. In: ESRI USER CONFERENCE, 21., San Diego. Proceedings...San Diego: ESRI, 2001.

PFAFSTETTER, O. Classificação de bacias hidrográficas. Rio de Jeneiro, RJ: Departamento Nacional de Obras de Saneamento, 1989. (Manuscrito não publicado)

RIBEIRO, C. A. A. S. et al. O desafio da delimitação de áreas de preservação permanente. Revista Árvore, v.29, n.2, p.203-212, 2005.

TONELLO, K. C. et al. Morfometria da bacia hidrográfica da Cachoeira das Pombas, Guanhães - MG. Revista Árvore, v.30, n.5, p.849-857, 2006.
SILVA, P. A. Classificação e codificação das bacias hidrográficas brasileiras segundo o Método Pfafstetter, com uso de geoprocessamento. In: ENCUENTRO DE LAS ÁGUAS, 2. 1999, . Montevideo. Anais... Montevideo: Governo do Uruguai/OEA/IIICA - Instituto Latino-americano de Cooperación para la Agricultura, 1999.

VERDIN, K. L.; VERDIN, J. P. A topological system for delineation and codification of the Earth's river basins. Journal of Hydrology, v.218, n.1/2, p.1-12, 1999. 
